\title{
Hepatitis-C- und HIV-Prävalenz sowie antiretrovirale Therapie in einem niederschwelligen Opiatsubstitutionsprogramm - Eine Querschnittsanalyse
}

\author{
P. Bruggmann ${ }^{1}$ \\ A. Seidenberg ${ }^{2}$ \\ D. Meili ${ }^{1}$ \\ B. Kesseli ${ }^{1}$ \\ M. Huber ${ }^{1}$
}

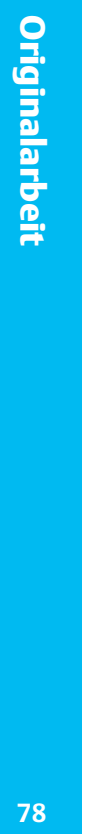

\author{
Hepatitis C - and HIV Prevalence and Antiretroviral Therapy in a Low Threshold \\ Opiate Maintenance Program
}

\section{Zusammenfassung}

Fragestellungen: Ist bei Patienten unter Opiatsubstitution im Vergleich zwischen 1997 und 2003 eine Abnahme der Prävalenzraten für Infektionen mit dem HI- beziehungsweise dem Hepatitis-C-Virus (HCV) zu beobachten? Sind antiretrovirale Therapien (ART) im Rahmen von Opiatsubstitutionsbehandlungen an einer Poliklinik ebenso effizient wie bei Patienten, die durch niedergelassene Ärzte mit Spezialisierung auf HIV betreut werden? Methode: In einer Querschnittsanalyse wurden im August 2003 erhobene serologische Befunde von Patienten eines Zürcher Opiatsubstitutionsprogramms vergleichbaren Daten einer Zürcher Studie aus dem Jahr 1997 gegenübergestellt. Die durchgeführten und erfolgreichen ART im Opiatsubstitutionsprogramm werden mit den statistischen Angaben von niedergelassenen Ärzten mit Spezialisierung auf HIV verglichen. Ergebnis: Der Vergleich der Jahre 1997 und 2003 zeigt eine signifikante Abnahme der HIV- (23,8\% vs. 9,6\% $\mathrm{p}<0,0001)$ und HCV-Prävalenz (57,8\% vs. 50,7\% p = 0,02) bei Patienten unter Opiatsubstitution. Bei HIV-Patienten unter Opiatsubstitution werden signifikant weniger ART duchgeführt als im Vergleichskollektiv der Patienten niedergelassener Ärzte (37\% vs. $62 \% \mathrm{p}=0,04)$. Die Therapieansprechraten sind in beiden Gruppen vergleichbar hoch. Schlussfolgerungen: Ein möglicher Grund für die sinkenden Prävalenzraten für HIV- und HCV-Infektionen unter Drogenabhängigen ist ein verändertes Risikoverhalten, insbesondere ein Rückgang des intravenösen Konsums. Unter den Bedingungen einer Opiatsubstitution ist es möglich, eine erfolgreiche antiretrovirale Therapie durchzuführen.

\section{Schliuisselwörter}

HIV · Hepatitis C · Prävalenz · Opiatsubstitution · Drogenkonsum

\section{Abstract}

Objective: Do the HIV and Hepatitis C (HCV) prevalence rates in participants of opiate maintenance programs decrease between 1997 and 2003? Do antiretroviral therapies (ART) in the setting of an opiate maintenance program show the same efficiency as in HIV positive patients attended by general practitioner? Study Design: In 2003 a cross sectional evaluation of HIV and HCV serology data in our opiate substitution program is done and compared with data of a study from 1997 in a similar setting. HIV positive patients under ART were assessed from clinical records and compared with the therapy rate and therapy efficacy (Viral load $<400 \mathrm{cpm}$ ) in the statistics of HIV specialised general practitioners. Results: There is a significant decrease in HIV $(23,8 \%$ vs. $9,6 \% \mathrm{p}<0.0001)$ and $\mathrm{HCV}(57,8 \%$ vs. $50,7 \% \mathrm{p}=0.02)$ prevalence between 1997 and 2003 in patients under opiate maintenance treatment. There are significantly less ART in the drug addicts group (37\%) as in the collective compared $(62 \% \mathrm{p}=0.04)$. The efficiency of the therapy was comparable. Conclusions: $A$ possible reason for the decreasing HIV and HCV prevalence could be changing risk behaviour. Under conditions of opiate substitution an effective ART is feasible.

\section{Key words}

HIV $\cdot$ hepatitis C . prevalence $\cdot$ opiate substitution · drug use

${ }^{1}$ Arbeitsgemeinschaft für risikoarmen Umgang mit Drogen, ARUD Zürich

${ }^{2}$ HIV Pract, Qualitätszirkel niedergelassener Ärzte in Zürich mit Schwerpunkt HIV-Behandlungen

Korrespondenzadresse

Dr. med. Philip Bruggmann · ARUD Zürich, Poliklinik für Drogenmedizin ZOKL1 · Sihlhallenstr. $30 \cdot 8004$

Zürich, Schweiz·E-mail: p.bruggmann@arud-zh.ch

Bibliografie

Suchttherapie 2005; 6: 78 -82 ๑ Georg Thieme Verlag KG Stuttgart · New York

DOI 10.1055/s-2005-858343

ISSN 1439-9903 
Die Neuinfektionsrate mit HIV durch intravenösen Drogenkonsum hat in den vergangenen Jahren in der Schweiz abgenommen [1]. Weniger eindeutig ist der Trend bezüglich Neuinfektionen mit dem Hepatitis-C-Virus; je nach Quelle wird in diesem Bereich von einer konstanten oder nur leicht rückläufigen Rate ausgegangen [2, 3]. Wie sich diese Entwicklung auf die Prävalenzraten in Opiatsubstitutionsprogrammen auswirkt, ist kaum untersucht worden. Wir gingen von der Hypothese aus, dass die Tendenzen, die bezüglich der Prävalenz von HIVund Hepatitis-C-Infektionen für Drogenkonsumenten insgesamt zu beobachten sind, auch im Rahmen von Opiatsubstitutionsprogrammen gelten.

Bezogen auf die Gesamtpopulation der Infizierten haben die antiretroviralen Behandlungen zu einer relevanten Reduktion von Morbidität und Mortalität der HIV-Infektion geführt [4]. Allerdings gibt es zunehmend Daten, die zeigen, dass die Reduktion der Mortalität bei Drogenabhängigen geringer ist als in anderen Subkollektiven [5]. Drogenabhängige gelten generell als schwierig zu behandelnde Patientengruppe [6], was der Vermutung Vorschub leistet, dass bei ihnen vergleichsweise wenige antiretrovirale Behandlungen durchgeführt werden. Es sind aber bisher kaum verlässliche Angaben darüber erhältlich, wie viele HIV-infizierte Drogenabhängige im Rahmen von OpiatSubstitutionsprogrammen tatsächlich antiretroviral behandelt werden. Eine Ausnahme bildet die 1997 durchgeführte so genannte Prometheus-Studie, an der vier Opiatabgabeinstitutionen in Zürich beteiligt waren. In dieser Studie wurden Kurzzeitdaten zur antiretroviralen Therapie im Rahmen von Drogensubstitutionsprogrammen erhoben [7]. Wie effektiv diese Behandlungen im Vergleich zu antiretroviralen Therapien sind, die bei Nichtdrogenabhängigen unter Alltagsbedingungen („Real life“) durch niedergelassene Ärzte durchgeführt werden, ist in Ermangelung aussagekräftiger Daten bisher nicht schlüssig zu beurteilen. Wir gingen von der Hypothese aus, dass abhängige HIV-Infizierte in Opiatsubstitutionsprogrammen ähnlich erfolgreich antiretroviral behandelt werden können wie andere Patientengruppen.

Mit der vorliegenden auf den Stichtag 15. August 2003 bezogenen Querschnittsanalyse von Daten einer Poliklinik zur opioidgestützten Behandlung drogenabhängiger Patienten in Zürich wurden folgende Ziele verfolgt:

- Erfassung der Prävalenz von HIV- und HCV-Infektionen in einem Opiatsubstitutionsprogramm im Jahr 2003;

- Erfassung der Häufigkeit einer HCV-PCR-Positivität bei HCVSeropositivität (persistierende HCV-Infektion);

- Vergleich der HIV- und HCV-Prävalenzen mit den Prävalenzraten, die 1997/98 im Rahmen der bereits erwähnten Prometheus-Studie erhoben wurden;

- Erfassung der Häufigkeit antiretroviraler Behandlungen sowie des Anteils der virologisch erfolgreichen Behandlungen;

- Vergleich von antiretroviraler Behandlungsfrequenz und -effektivität mit den entsprechenden Daten eines Kollektivs von HIV-Infizierten, die zum Untersuchungszeitpunkt von niedergelassenen, auf HIV spezialisierten Ärzten behandelt wurden.

\section{Behandlungszentrum}

An der Poliklinik für Drogenmedizin ZOKL1 der ARUD Zürich werden Patientinnen und Patienten im Rahmen von Opiatsubstitutionsprogrammen nach den kantonalen Richtlinien mit Methadon oder Buprenorphin behandelt [8]. Es erfolgt eine hausärztliche Versorgung der Patienten mit einem Schwerpunkt auf der infektiologischen Betreuung, insbesondere in den Bereichen HIV und virale Hepatitiden.

\section{Messparameter}

Für Patientinnen und Patienten gelten an der Poliklinik für Drogenmedizin ZOKL1 bezüglich der Erfassung und Behandlung von Infektionskrankheiten - speziell HIV- und virale Hepatitiden definierte Standards. Diese gelangten auch bei den im Rahmen der Querschnittsuntersuchung erfassten Patienten zur Anwendung und werden im Folgenden kurz beschrieben.

Allen behandelten Patienten werden ein Anti-Hepatitis-C- und HIV-Antikörpertest sowie bei Positivität je die entsprechenden PC-Reaktionen (Nachweis viraler Erbsubstanz) angeboten. Die Blutentnahmen finden an der Poliklinik für Drogenmedizin statt; die Laboruntersuchungen werden in den Laboratorien des Universitätsspitals Zürich durchgeführt. Bei einer HIV-Infektion werden regelmäßig immunologische und virologische Parameter untersucht. Falls diese Untersuchungen länger als 6 Monate zurückliegen, werden sie für die Messparameter „aktuelle Werte“ als unbekannt klassiert. Bei gegebener Indikation wird den HIV-infizierten Patienten eine antiretrovirale Behandlung angeboten, welche gemäß den aktuell gültigen Richtlinien [9] an der Poliklinik für Drogenmedizin durchgeführt wird. Die Abgabe der antiretroviralen Medikamente erfolgt meistens im Rahmen der Methadonabgabe. Die Poliklinik war zum Erhebungszeitpunkt Teilnehmerin an der schweizerischen HIV-Kohortenstudie. Die Behandlungen und deren Kontrollen werden handschriftlich und elektronisch in den entsprechenden Krankengeschichten festgehalten.

Die Seroprävalenzen bezüglich HIV- und HCV-Infektionen wurden aus der hausinternen Datenbank am Stichtag 15.8.2003 erhoben. Weitere Laborwerte und die Anamnese bezüglich antiretroviraler Behandlung wurden für den gleichen Zeitpunkt den Krankengeschichten entnommen.

\section{Vergleiche}

Die Vergleiche der Seroprävalenzen erfolgten mit Prävalenzdaten von 1997/1998 aus der Prometheusstudie, an der vier Zürcher Drogensubstitutionseinrichtungen - darunter auch die Poliklink ZOKL1 - beteiligt waren [7]. Die serologischen Parameter im Rahmen dieser Studie wurden ebenfalls in den Laboratorien des Universitätsspitals Zürich ermittelt. Die Häufigkeit antiretroviraler Behandlung zum Erhebungszeitpunkt, die Häufigkeit früherer antiretroviraler Behandlungen, die Häufigkeit der Koinfektion mit HCV und der Anteil virologisch erfolgreicher antiretroviraler Therapien (HIV PCR unter 50 resp. 400 Viruskopien pro $\mathrm{ml}$ ) wurden mit bis anhin nicht publizierten Daten von HIV Pract verglichen, einer Vereinigung von niedergelassenen Ärztinnen und Ärzten, die im Raum Zürich ambulante Betreuungen und Behandlung von HIV-Infizierten durchführen [10]. 


\section{Statistik}

Die Zielgrößen beziehungsweise deren Anteile in den verschiedenen Datensammlungen wurden mittels etablierter statistischer Verfahren bestimmt [11]. Unterschiede bezüglich Merkmalshäufigkeiten wurden mittels des $\chi^{2}$-Tests berechnet. Die 95\%-Vertrauensintervalle (VI) der erhobenen Größen wurden mit Mittelwert plus/minus 2 Standardfehlern angegeben.

\section{Ergebnisse}

\section{HIV-Prävalenz (Abb.1)}

Am Stichtag 15. August 2003 wurden in der Poliklinik ZOKL1 522 Patientinnen im Rahmen von Opiatsubstitutionsprogrammen 503 mit Methadon, 15 mit Buprenorphin - behandelt. Allen Behandelten war ein HIV-Test angeboten worden; zum Erhebungszeitpunkt lagen bei 397 von 522 Behandelten valide HIV Serologien vor (76\%). 38-mal war die konfirmierte Serologie positiv (9,6\% der Getesteten), einmal lag eine nicht bestätigte positive Serologie vor (wahrscheinlich aufgrund einer Blutverwechslung). In der Prometheusstudie 1997/98 lagen 499 Testresultate von 603 Klienten vor (83\%); auch in diesem Fall war ein Test allen Patienten angeboten worden. 119-mal wurde eine positive Serologie getestet (23,8\%). Der Unterschied bezüglich der Testfrequenz ist nicht signifikant; der Unterschied bezüglich der Seropositivität ist mit p kleiner als 0,001 hochsignifikant.

\section{HCV-Serologie/HCV-PCR (Abb. 2)}

Sowohl im Rahmen der Opiatsubstitutionsbehandlung in der Poliklinik ZOKL1 als auch im Rahmen der Prometheus-Studie wurden allen Patienten Tests zur Erfassung einer HCV-Infektion beziehungsweise des Immunstatus angeboten. Am 15. August 2003 lag bei 381 von 522 Patienten der Poliklinik ZOKL1 ein valides Ergebnis einer Hepatitis-C-Serologie vor (73\%). Die Serolo-

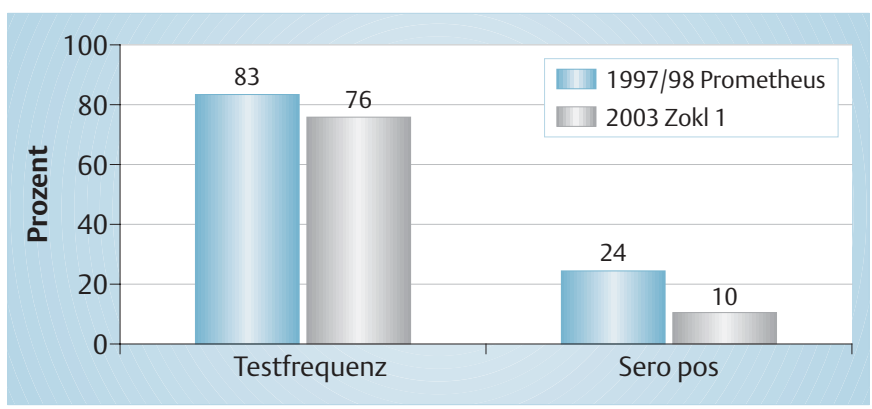

Abb. 1 Vergleich der HIV-Prävalenzdaten aus Opiatsubstitutionskliniken in Zürich. Sero pos = HIV-Serologie positiv.

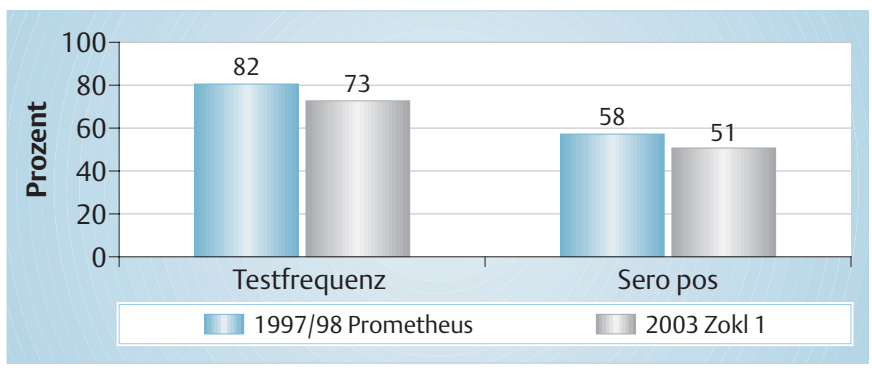

Abb. 2 Vergleich der Hepatitis-C-Prävalenzdaten aus Opiatsubstitutionskliniken in Zürich. Sero pos = Hepatitis-C-Serologie positiv . gie war 193-mal positiv (50,7\%). In der Prometheus-Studie lag bei 211 von 496 Getesteten (57,8\%) eine Seropositivität für HCV vor. Die Abnahme der Seroprävalenz ist mit einem p von 0,02 signifikant.

Am 15. August 2003 lag bei 119 der 193 HCV-Serologie-Positiven eine HCV-PCR vor, welche 73-mal positiv und 46-mal (38,7\%, VI 29 bis $47 \%$ ) negativ war. In der Prometheus-Studie wurde die HCV-PCR nicht untersucht.

\section{HIV-Infizierte: Basisdaten (Tab. 1)}

Das Durchschnittsalter der in der Poliklinik ZOKL1 behandelten HIV-Infizierten lag am 15. August 2003 bei 37 Jahren (Standardabweichung 6,8 Jahre, Range 21 bis 52 Jahre). Die Seropositivität war im Durchschnitt 6 Jahre bekannt (Standardabweichung 4,3 Jahre, Range 0 bis 17 Jahre). 12 der 38 HIV-Infizierten waren Frauen (31,5\%). CD4-Zellwerte lagen bei 31 von 38 Patienten vor, die tiefsten je gemessenen CD4-Zellen (Nadir) lagen im Durchschnitt bei 240 (Standardabweichung 173 Zellen, Range 3 bis 557 Zellen). Der Durchschnittwert des höchsten je gemessenen HIV-PCR-Wertes lag bei 4,9 Log ( $\pm 0,8 \log )$. Gemäß den Kriterien der schweizerischen HIV-Kohortenstudie lag 15-mal ein Stadium A gemäß CDC vor, 12-mal ein Stadium B, 3-mal ein Stadium C. 10-mal ließ sich wegen fehlender Angaben keine eindeutige Klassierung vornehmen. Bei 32 von 38 Patienten lag eine positive HCV-Serologie vor. Mit $84 \%$ war dieser Anteil hochsignifikant häufiger als im Vergleichskollektiv von HIV Pract, in dem der Prozentsatz der Seropositiven 35\% betrug. 17-mal war die HCVPCR positiv, 7-mal negativ (davon einmal nach Interferon-/Ribavirintherapie), 8-mal wurde keine Hepatitis-C-PCR durchgeführt. Alle Patienten waren in Opiatsubstitution (HIV Pract: 23\%). 16 der 38 erfassten Klienten waren Teilnehmer der schweizerischen HIV-Kohortenstudie.

\section{Antiretrovirale Behandlungen: Frequenz (Tab. 2)}

14 von 38 HIV-Infizierten (37\%) waren am Stichtag 15. August 2003 in antiretroviraler Behandlung. Im HIV-Pract-Kollektiv wurden zu diesem Zeitpunkt 316 von 508 Patienten $(62,2 \%)$ mit antiretroviralen Medikamenten behandelt. Der zwischen den Vergleichskollektiven festgestellte Unterschied bezüglich des Anteils behandelter Patienten ist signifikant $(p=0,04)$. Der Durchschnitt der Behandlungsdauer betrug im Fall der Poliklinik ZOKL1 42 Monate (Standardabweichung 34 Monate). Angaben zur Behandlungsdauer im HIV-Pract-Kollektiv lagen nicht vor. Bei 8 der aktuell antiretroviral Behandelten wurde noch mit der Ersttherapie behandelt, bei 6 wurde vorhergehend mindestens

Tab. 1 Basiseigenschaften der HIV-Infizierten

\begin{tabular}{lll}
\hline & HIV Pract & ZOKL1 \\
& & \\
\hline $\mathrm{n}$ & 508 & 38 \\
\hline unter Opiatsubstitution & $118(23 \%)$ & $38(100 \%)$ \\
\hline HCV-seropositiv & $179(35 \%)$ & $32(84 \%)$ \\
\hline weiblich & $151(30 \%)$ & $12(31 \%)$ \\
\hline
\end{tabular}

${ }^{1}$ HIV Pract: HIV-Patienten des Qualitätszirkels HIV-spezialisierter praktizierender Ärzte, Zürich

2 ZOKL1: HIV-Patienten der Poliklinik für Drogenmedizin mit Opiatsubstitution, ZOKL1, ARUD Zürich 
Tab. 2 Antiretrovirale Behandlungen (ART)

\begin{tabular}{llll}
\hline & HIV Pract $^{1}$ & ZOKL1 $^{2}$ & P Value \\
\hline $\mathrm{n}$ & 508 & 38 & \\
\hline aktuell in ART & $316(62 \%)$ & $14(37 \%)$ & 0,04 \\
\hline nie ART & $88(17 \%)$ & $18(47 \%)$ & 0,07 \\
\hline virologischer Erfolg & 257 von $316(81 \%)$ & 11 von $14(79 \%)$ & nicht signifikant \\
\hline
\end{tabular}

${ }^{1}$ HIV Pract: HIV-Patienten des Qualitätszirkels HIV-spezialisierter praktizierender Ärzte, Zürich

2 ZOKL1: HIV-Patienten der Poliklinik für Drogenmedizin mit Opiatsubstitution, ZOKL1, ARUD Zürich

ein weiteres Regime durchgeführt. Bei 6 weiteren, aktuell nicht behandelten Patienten wurde früher eine antiretrovirale Therapie durchgeführt, welche - aus unterschiedlichen Gründen - abgebrochen wurde. 18 Patienten (47,4\%) hatten noch nie eine antiretrovirale Therapie erhalten. Im HIV-Pract-Kollektiv betrug der entsprechende Anteil 20\%, der Unterschied lag knapp außerhalb der Signifikanzgrenze $(p=0,07)$.

\section{Antiretrovirale Behandlungen: Effektivität (Tab. 2)}

Von den 14 antiretroviral Behandelten der Poliklinik ZOKL1 hatten am Stichtag 15. August 20038 eine HIV-PCR von unter 50 Viruskopien pro $\mathrm{ml}$ (Anteil: 57\%). 3 weitere hatten eine HIV-PCR unter 400 - teils noch in der frühen Behandlungsphase. Insgesamt waren 11 von 14 Behandelten (78\%) mit einem Viral Load von unter 400 zu verzeichnen. Im HIV-Pract-Kollektiv betrug der entsprechende Anteil $81 \%$ der Unterschied zum ZOKL1-Kollektiv ist nicht signifikant.

\section{Diskussion}

Mit der Querschnittsanalyse von Daten der Opiatsubstitutionspoliklinik ZOKL1 in Zürich sowie dem Vergleich dieser Daten mit früheren Erhebungen im Rahmen der so genannten PrometheusStudie konnten wir zeigen, dass die Prävalenz der HIV-Infektion bei Opiatsubstituierten von 1998 bis 2003 signifikant abgenommen hat. Zudem stellten wir eine geringe, aber signifikante Reduktion der Hepatitis-C-Seroprävalenz fest.

Der Vergleich unserer Daten mit bisher nicht publizierten Zahlen zur HIV-Behandlung durch niedergelassene Ärzte zeigte, dass die in der Poliklinik für Drogenmedizin ZOKL1 betreuten Patienten signifikant häufiger mit Hepatitis C koinfiziert sind, zum Erhebungszeitpunkt signifikant seltener antiretroviral behandelt wurden und häufiger noch nie antiretroviral behandelt wurden.

Der Behandlungserfolg nach 42 Monaten antiretroviraler Therapie, gemessen an der HI-Viruslast, ist annähernd identisch mit der Erfolgsrate im Vergleichskollektiv der HIV-Pract-Ärzte. Vergleichbare Zahlen werden in der Literatur auch für weitere, unter „Real-life-Bedingungen“ studierte Patientengruppen genannt [12].
Tab. 3 IV-Drogenkonsum der ZOKL-1-Patienten 1992-2002 (institutionsinterne Statistik)

\begin{tabular}{lcccccc}
\hline & 1992 & $\begin{array}{l}1993 \\
1994\end{array}$ & $\begin{array}{l}1995 \\
1996\end{array}$ & $\begin{array}{l}1997 \\
1998\end{array}$ & $\begin{array}{l}1999 \\
2000\end{array}$ & 2001 \\
\hline $\begin{array}{l}\text { Männer } \\
\%\end{array}$ & 61,6 & 51,3 & 45,3 & 47,1 & 48,8 & 43,9 \\
$\begin{array}{l}\text { IVDU } \\
\text { Frauen } \\
\%\end{array}$ & 64,9 & 49,7 & 43,5 & 38,8 & 47,9 & 42,4 \\
IVDU & & & & & & \\
\hline n tot & 634 & 539 & 308 & 291 & 243 & 278 \\
\hline
\end{tabular}

IVDU: intravenöser Drogenkonsum

Auf Hepatitis-C-Behandlungen an den Drogenpolikliniken der ARUD Zürich wird in diesem Artikel nicht näher eingegangen. Diese Therapien werden bei allen Patienten mit positiver HCVSerologie evaluiert und angeboten [13].

Die festgestellten Abnahmen von HIV- und HCV-Prävalenzen in Opiatsubstitutionsprogrammen sind mit gewissen Einschränkungen zu beurteilen. Die Daten von 1997/98 stammen aus ähnlichen, aber nicht den gleichen Institutionen, so dass ein gewisser Rekrutierungsbias nicht auszuschließen ist. Ob die Reduktion auf verändertes Risikoverhalten - beispielsweise den rückläufigen intravenösen Drogenkonsum (Tab.3) - oder auf verändertes Beanspruchen der Substitutionsprogramme durch infizierte Abhängige zurückzuführen ist und welchen Einfluss die durch die Behandlung veränderten Überlebensraten auf die Prävalenzraten ausüben, kann aufgrund der vorliegenden Daten nicht schlüssig beantwortet werden.

Die Zahlen zur Behandlungsfrequenz und -effektivität entstammen einer kleinen Datenbasis und haben somit ein großes Vertrauensintervall. Auch ist das hier untersuchte Kollektiv nicht direkt vergleichbar mit den in den Statistiken von HIV Pract erfassten Patienten. Trotzdem kann der Anteil der virologisch erfolgreich behandelten Patienten als erfreulich hoch bezeichnet werden. Dieses Ergebnis kann als Hinweis darauf interpretiert werden, dass unter den Bedingungen eines Opiatsubstitutionsprogramms bei einem Teil der Substituierten nicht nur im Rahmen von Studien, sondern auch unter "Real-life-Bedingungen“ eine erfolgreiche antiretrovirale Therapie durchgeführt werden kann, wenn am Zentrum das entsprechende Fachwissen vorhanden ist.

\section{Literatur}

${ }^{1}$ Bundesamt für Gesundheit (BAG). Infektionskrankheiten, Schweiz 2002. www.bag.admin.ch/infreporting/gs02/p99.pdf

2 Steffen T, Gutzwiller F. Hepatitis B und C bei intravenös Drogenkonsumierenden in der Schweiz. Praxis 1999; 88: 1937-1944

3 Bundesamt für Gesundheit (BAG). Infektionskranhkeiten, Schweiz 2002. www.bag.admin.ch/infreporting/gs02/p19.pdf

${ }^{4}$ Egger M, Hirschel B, Francioli P et al. Impact of new antiretroviral combination therapies in HIV infected patients in Switzerland: prospective multicentre study. Swiss HIV Cohort Study. BMJ 1997; 315: $1194-1199$

${ }^{5}$ Moore RD, Keruly JC, Chaisson RE. Differences in HIV disease progression by injecting drug use in HIV-infected persons in care. J Acquir Immune Defic Syndr 2004; 35: 46-51 
${ }^{6}$ Bouhnik AD, Chesney M, Carrieri P et al. Nonadherence among HIV-infected injecting drug users: the impact of social instability. J Acquir Immune Defic Syndr 2002; 31 (Suppl 3): 149-153

${ }^{7}$ Flepp M. PROMETHEUS, Projekt zur Abschätzung von Bedürfnis, Machbarkeit, Wirkung und Compliance einer antiretrovirealen HIV Therapie bei DrogenbenützerInnen. AIDS Forschung Schweiz, 2000: 26

${ }^{8}$ Direktion des Gesundheitswesens des Kantons Zürich, 1996: Richtlinien zur methadonunterstützten Behandlung Heroinabhängiger vom 20. März 1996. Gesundheitsdirektion Zürich, 1996

${ }^{9}$ Subkommission Klinik der eidgenössischen Kommission für Aids-Fragen: Empfehlungen zur antiretroviralen HIV-Therapie 2001. BAG Bulletin 2000; 51: $994-1001$
${ }^{10}$ HIV Pract, Qualitätszirkel praktizierender ÄrztInnen, Zürich. www.hiv-pract.ch. Unpublizierte Daten der Jahresstatistiken.

${ }^{11}$ Glantz S. Biostatistik. Mit begleitendem PC Programm. Frankfurt: Mc Graw Hill, 1998

12 Wood E, Montaner JS, Yip B et al. Adherence and plasma HIV RNA responses to highly active antiretroviral therapy among HIV-1 infected injection drug users. CMAJ 2003; 169: 656-661

${ }^{13}$ Huber M, Schmid P, Vernazza P et al. Gründe für die ausbleibende Hepatitis-C-Behandlung bei Drogenabhängigen in Opiatsubstitution. Suchttherapie 2002; 3: $27-30$

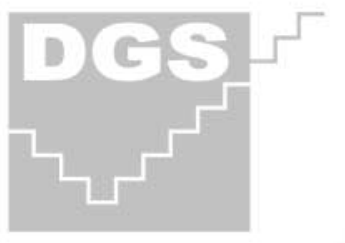

\section{Deutsche Gesellschaft für Suchtmedizin (vorm. DGDS) e.V. 14. Suchtmedizinischer Kongress}

"Qualität und Individualität in der Suchtmedizin“ 04. - 06. November 2005 in Berlin

Symposien zu den Themenkreisen Qualität und Individualität in der Suchtmedizin Sucht und schwere Komorbidität Versorgung und Kooperationsformen

\section{Kontakt und weitere Informationen:}

DGS Geschäftsstelle

Frau Maren Stich

c/o Zentrum für Interdisziplinäre Suchtforschung (ZIS) der Universität Hamburg

Martinistrasse 52

20246 Hamburg

Telefon/Fax: +49 40428035121

Email: kongress@dgsuchtmedizin.de

Home: www.dgsuchtmedizin.de 